\section{The Proportional Navigation Dilemma-Pure or True?}

\author{
U. S. SHUKLA \\ I? R. MAHAPATRA \\ Indian Institute of Science \\ India
}

Two generic classes of proportional navigation (PN) laws are compared in detail. One class consists of pursuer velocity referenced system which includes pure proportional navigation $(\mathrm{PPN})$ and its variants and the second category consists of line-of-sight (LOS) referenced system such as true proportional navigation (TPN), generalized true proportional navigation (GTPN) and generalized guidance laws. The existing closed-form solutions are discussed in detail while summarizing the classical linear and quasilinear analytical solutions. A critical comparison is then made with regard to their definition, implementation, analytical aspects including the method and the nature of solution, and more importantly, an appraisal of the behavior of the pursuer motion resulting from these guidance laws. It is established that in spite of some restricted advantages in the solvability of the equations of motion, the LOS-referenced PN schemes suffer from serious limitations in term of implementation and trajectory behavior. Among the major drawbacks are forward velocity variation requirement (requiring use of thrusters; implementation not possible in aerodynamically controlled pursuers), relatively large control effort requirement, restrictions on initial engagement condilions to ensure intercept, lack of robuslness and possibility of unbounded acceleration. This leads to the firm conclusion that PPN is the nore "natural" guidance law in a practical sense as compared with TPN and its generalizations. Thus, although most of the analytical effort hitherto appears to have been concentrated on TPN and its generalizations, more serious effort needs to be made to understand, model, and solve the PPN guidance scheme.

Manuscript received May 8, 1989; revised September 18, 3989. IEEE Log No. 33465.

Authors' address: Department of Aerospace Engineering, Indian Institute of Science, Bangalore 560012, India.

0018-9251/90/0300-0382 \$1.00 (C) 1990 IEEE

\section{Variables}

$\boldsymbol{A}$ Lateral Acceleration.

$N \quad$ Navigation constant.

$N^{\prime} \quad$ Effective navigation constant.

$\boldsymbol{V} \quad$ Velocity.

AV Cumulative velocity increment.

$r \quad$ Range from pursuer to target.

$t \quad$ Time.

$\phi \quad$ Angle between pursuer velocity vector and reference line.

$\Delta \phi \quad$ Heading error relative to collision course.

$\psi \quad$ Pursuer heading angle.

$\theta \quad$ Angle of line of sight (LOS) relative to reference line.

$P \quad$ Angle between target velocity vector and reference line.

$\Delta \beta \quad$ Incremental rotation of target velocity vector from its initial direction.

$\kappa \quad$ Normal turn rate of the target.

\section{Subscripts}

$M \quad$ Pursuer.

PPN Pure PN.

TPN True PN.

$T$ Target.

1 Initial values.

$f \quad$ Final value (at intercept).

$R \quad$ Relative closing (velocity) along line of sight.

$\boldsymbol{R} \boldsymbol{i} \quad$ Initial relative closing (velocity) along

line of sight.

$\theta \quad$ Normal to line of sight.

$\theta_{i} \quad$ Initial normal to line of sight.

$\phi \quad$ Normal to pursuer velocity vector.

\section{INTRODUCTION}

Many of the currently operational tactical guided missiles employ proportional navigation $(\mathrm{PN})$ as the guidance law for terminal guidance. Surface-to-air, air-to-air, and air-to-surface missile engagements, "stand off" weapon delivery as well as space applications such as rendezvous use PN in one form or another as a guidance law [1]. For an aerodynamically controlled missile the PN law may be considered as the optimal pursuit strategy in the sense of minimizing the terminal miss distance [2].

A major advantage of PN, contributing to its durability as a favored guidance scheme during the last few decades, is its relative simplicity of implementation in practical systems. For implementation, it requires low levels of information input regarding the target characteristics (including motion) compared with many other more elaborate schemes, thus simplifying onboard sensor requirements and improving reliability 
and robustness. The scheme is based entirely on the instantaneous direction of the target relative to the pursuer in space, and its first derivative with respect to time (a second derivative may be utilized in certain augmented PN schemes). These quantities are easily sensed using active or passive microwave, infrared, optical or acoustic trackers, and the basic PN scheme consists in applying to the pursuer a control force (through control surface deflections or control thruster operation) proportional to the rate of rotation of the line of sight (LOS) and acting along a direction defined by the specific variant of PN employed.

In view of the importance of the PN law in the missile guidance and space applications, considerable analytical study has been made regarding the behavior of projectiles under the PN pursuit law. Since the differential equations governing PN motion, even considering kinematics only, are highly nonlinear, only limited success has been achieved in solving these equations. In attempting to solve the PN equations, approaches have been made hitherto along two principal directions. The prime difference between these two directions arises from the way PN is defined. While one set of definitions leads to a class of laws consisting of pure proportional navigation (PPN) and its variants, which are referenced relative to the pursuer velocity vector, the other class has LOS referenced laws like the true proportional navigation (TPN) and its generalizations as its members.

From the point of view of mathematical analysis, it has been possible to solve the LOS-referenced laws in closed form (though the solutions are mostly implicit in nature) for at least the nonmaneuvering target case. In contrast, PPN has been solved in closed form, for the nonmaneuvering target case, only for the less useful discrete values 1 and 2 of $N$, the navigation constant. It would thus appear that the TPN has an edge over PPN in terms of mathematical tractability. This may be the reason that a number of papers have appeared in the literature dealing with the solution of the TPN problem [3] and its generalizations such as the generalized true proportional navigation (GTPN)

$[4,5]$ and the generalized guidance law [6].

It is shown in this paper that notwithstanding the relative difficulties in solving the PPN problem, PPN is the most "natural" PN law. This conclusion is drawn based on the facts that the LOS-referenced laws are not practically implementable, require forward acceleration/deceleration, are inefficient in terms of control effort, lead to severe constraints on the initial engagement geometry to ensure acceptable trajectory behavior and intercept and are thus less robust. Also, the relative analytical advantage of LOS-referenced PN laws does not appear sustainable as more realistic PN scenarios are analyzed.

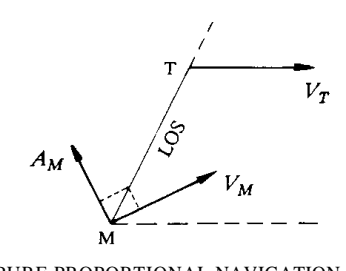

PURE PROPORTIONAL NAVIGATION

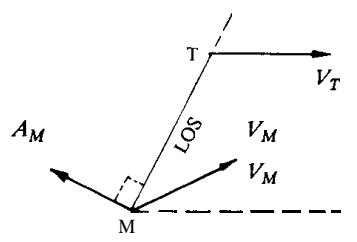

TRUE PROPORTIONAL NAVIGATION

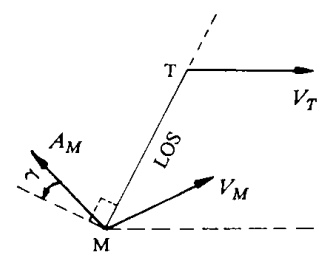

GENERALIZED TRUE PROPORTIONAL NAVIGATION

Fig. 1. Geometries of the three variants of $\mathrm{PN}$.

\section{DEFINITIONS}

Early studies of PN $[7,8]$ treat the PN strategy as one in which the guided point (pursuer) moves towards a target point in a plane containing the velocity vectors of the two points, and define the strategy such that the velocity vector (heading) of the pursuer is rotated at a rate proportional to the rotation rate of the line joining the pursuer and the target (line of sight). A later development in the PN definition has been to make the lateral acceleration of the pursuer vary in proportion with the rate of rotation of the LOS.

Although the basic PN principle establishes the magnitude of the lateral acceleration (or control force) in relation to the LOS turn rate, slightly different definitions of $\mathrm{PN}$ are possible depending on the direction of application of this acceleration/force. Three different types of PN $[3,4,9]$ are defined: the PPN, the TPN, and the GTPN.

In PPN, the desired lateral acceleration (proportional to LOS turning rate) is applied normal to the velocity vector of the pursuer. In TPN, the control force is applied normal to the instantaneous LOS. Finally in GTPN, the missile acceleration is not necessarily applied normal to the LOS but must have a fixed angle relative to it. The three schemes are as shown in Fig. 1. 


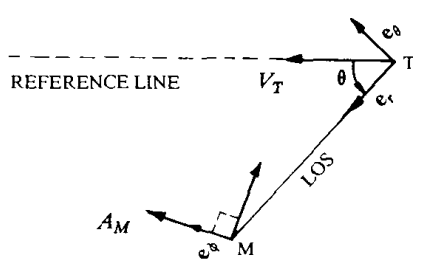

PURE PROPORTIONAL NAVIGATION

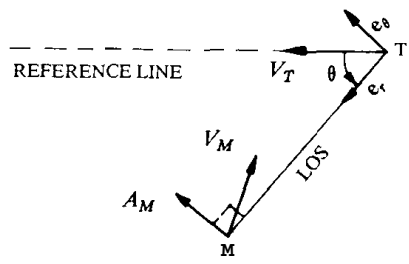

TRUE PROPORTIONAL NAVIGATION

Fig. 2. Target-pursuer vector geometry.

The equations of motion of the pursuer are written in vector form as

$$
\mathbf{F}=\boldsymbol{m} \frac{d^{2} \mathbf{r}}{d t^{2}}
$$

As shown in Fig. 2, a unit vector triad $\left(\mathbf{e}_{r}, \mathbf{e}_{\theta}, \mathbf{e}_{k}\right)$ is constructed with its origin at the target, $\boldsymbol{e}$, aligned with the LOS and $\mathbf{e}_{\boldsymbol{k}}$ normal to the plane of vehicle velocity vectors. The rotation of $\left(\mathbf{e}_{r}, \mathbf{e}_{\theta}, \mathbf{e}_{k}\right)$ triad with respect to an inertial reference (taken as the initial direction of target motion) is

$$
\Omega=\theta \mathbf{e}_{k} .
$$

Then, from kinematics of a point in a rotating frame

$$
\begin{aligned}
\mathbf{r} & =r \boldsymbol{e}, \\
\frac{d \boldsymbol{r}}{d t} & =r e,+\Omega x \mathbf{r} \\
& =\dot{r} \mathbf{e}_{r}+r \dot{\theta} \mathbf{e}_{\theta} \\
\frac{d^{2} \mathbf{r}}{d t^{2}} & =\ddot{r} \mathbf{e}_{r}+\Omega \times \mathbf{r}+\Omega \times(\Omega \times \mathbf{r})+2 \Omega \times \mathbf{r} \\
& =\left(\ddot{r}-r \dot{\theta}^{2}\right) \mathbf{e}_{r}-(r \ddot{\theta}+2 \dot{r} \dot{\theta}) \mathbf{e}_{\theta} .
\end{aligned}
$$

$\mathbf{F}$, the applied force on the pursuer, is assumed to be the only control force.

Considering TPN first, and resolving the applied force along and normal to the LOS

$$
\begin{aligned}
A_{M} & =\frac{\mathbf{F}}{m}=F_{\theta} \mathbf{e}_{\theta}+F_{r} \mathbf{e}_{r} \\
& =\boldsymbol{\Lambda}_{\theta} \theta \mathbf{e}_{\theta}+\boldsymbol{\Lambda}_{r} \theta \mathbf{e}_{r},
\end{aligned}
$$
using the definition of PN.

For GTPN, if $\gamma$ is the constant angle between the direction of the applied pursuer acceleration and the direction normal to the LOS, then [4]

$$
\mathbf{A},=\lambda V_{\theta_{i}} \sin \gamma \quad \text { and } \quad \boldsymbol{\Lambda}_{\theta}=-\lambda V_{R i} \cos \gamma
$$

where the subscript $i$ denotes the initial value and $\lambda$ is the navigation constant.

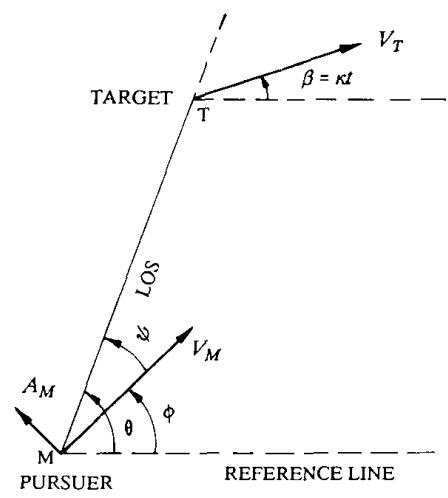

Fig. 3. Geometry of planar pursuit against maneuvering target for PPN.

For TPN, $\gamma=0$ and hence

$$
\begin{gathered}
\Lambda_{r}=0 \quad \text { and } \quad \Lambda_{\theta}=-\lambda V_{R i} \\
A_{M \text { (TPN) }}=-\lambda V_{R i} \dot{\theta} \mathbf{e}_{\theta} .
\end{gathered}
$$

Next, considering PPN, the control force is applied normal to the pursuer velocity vector and hence

$$
A_{M(\mathrm{PPN})}=\frac{\mathbf{F}}{m}=V_{M} \dot{\phi} \mathbf{e}_{\phi}
$$

where 6 , is the pursuer turn rate. From the definition of $\mathrm{PN}$

$$
A_{M(\mathrm{PPN})}=N V_{M} \dot{\theta} \mathbf{e}_{\phi} .
$$

Using (12) and (13)

$$
\dot{\phi}=N \dot{\theta} \text {. }
$$

Equation (14) corresponds to the original natural definition of PPN relating the pursuer turn rate to the LOS rate through a constant [7].

Having outlined two basic ways of defining the PN law, one through the desired pursuer turn rate and the other through the applied control force, and having shown the equivalence of the two for the PPN strategy, we now proceed to review the mathematical basis of the formulation for the various types of PN.

\section{PURE PROPORTIONAL NAVIGATION}

We consider the general case of a planar pursuit against a maneuvering target depicted in Fig. 3. The target $T$ and the pursuer $M$ are considered to be geometric points with constant velocities $V_{T}$ and $V_{M}$ and normal accelerations $A_{T}$ and $A_{M}$, respectively. The pursuit is described in a coordinate system centered at $T$ and with respect to a reference line parallel to the initial direction of $V_{T}$. The target acceleration $A_{T}$ is assumed constant.

Resolving the target velocity $V_{T}$ and the pursuer velocity $V_{M}$ along and normal to the LOS, we obtain

$$
\dot{r}=V_{T} \cos (\theta-\beta)-V_{M} \cos (\theta-\phi)
$$


and

$$
r \dot{\theta}=-V_{T} \sin (\theta-\beta)+V_{M} \sin (\theta-\phi)
$$

where

$$
\begin{aligned}
& \beta=\kappa t \\
& \kappa=A_{T} / V_{T}
\end{aligned}
$$

and $\kappa$ represents the normal turn rate of the target. The definition (14) of PPN, i.e. $\dot{\phi}=N \dot{\theta}$, can be integrated to give

$$
\theta-\phi=b \theta-c
$$

where

$$
b=1-N \quad \text { and } \quad c=\phi_{i}-N \theta_{i}
$$

Substituting (18) in (15) and (16), we obtain

$$
\dot{r}=V_{T} \cos (\theta-\beta)-V_{M} \cos (b \theta-c)
$$

and

$$
r \dot{\theta}=-V_{T} \sin (\theta-\beta)+V_{M} \sin (b \theta-d .
$$

Defining the radial (i.e., along LOS) and normal components of the pursuer-target relative velocities as

$$
V_{R}(\theta, t)=V_{T} \cos (\theta-\beta)-V_{M} \cos (b \theta-c)
$$

and

$$
V_{\theta}(\theta, t)=-V_{T} \sin (\theta-\beta)+V_{M} \sin (b \theta-c)
$$

(20) and (21) can be written, respectively, as

$$
\dot{r}=V_{R}(\theta, t)
$$

and

$$
\boldsymbol{r e}=V_{\theta}(\theta, t)
$$

For the nonmaneuvering target case $\left(A_{T}=0\right),(20)$ and (21) reduce to

$$
\dot{r}=V_{T} \cos \theta-V_{M} \cos (b \theta-c)
$$

and

$$
r \dot{\theta}=-V_{T} \sin \theta+V_{M} \sin (b \theta-c)
$$

respectively. Here, the radial and normal components of the relative velocity can be defined as

$$
\dot{r}=V_{R}(\theta)
$$

and

$$
r \dot{\theta}=V_{\theta}(\theta)
$$

where

$$
V_{R}(\theta)=V_{T} \cos \theta-V_{M} \cos (b \theta-c)
$$

and

$$
V_{\theta}(\theta)=-V_{T} \sin \theta+V_{M} \sin (b \theta-c) .
$$

The system of equations (24) and (25) describing the pursuit against a general case of maneuvering target are highly nonlinear. No closed-form solutions are available for these equations for any value of the navigation constant $N$. These general equations reduce to (28) and (29) for the case of a nonmaneuvering target. Although there is still no closed-form solution available even for this simpler case for general values of $N$, exact solutions have been obtained in the literature [7] for the two particular values of $N=1$ and $N=2$ only. These solutions are discussed in the following section.

A. Special Analytical Solutions of Pure Proportional Navigation

For the case of a nonmaneuvering target, dividing (28) by (29)

$$
\frac{1}{r} \frac{d r}{d \theta}=\frac{V_{R}(\theta)}{V_{\theta}(\theta)}
$$

On integrating, (32) yields

$$
r=r_{i} \exp \left[\int_{\theta_{i}}^{\theta} \frac{V_{R}}{V_{\theta}} d \theta\right] .
$$

Equation (33) provides $r$ as a function of $\theta$. The integral appearing here, with $V_{R}$ and $V_{\theta}$ replaced from (30) and (31), respectively, is not solvable in general. It can be performed only in two particular cases: $N=\mathbf{1}$ and $N=2$.

The case $N=1$, in fact, reduces PPN to the simple pursuit course: deviated pursuit in general and pure pursuit when $\phi_{i}=\theta_{i}$, i.e., when the pursuer velocity vector is initially directed toward the target position. For simplicity, we quote the solution for the pure pursuit course only [7]:

$$
r=\frac{r_{i}\left(\sin \theta_{i}\right)^{p+1}}{\left(1+\cos \theta_{i}\right)^{p}} \frac{(1+\cos \theta)^{p}}{(\sin \theta)^{p+1}}
$$

where $p=V_{M} / V_{T}$.

The solution corresponding to $N=2$ has been derived by Locke [7] in terms of the angle $\psi$ between the LOS and the pursuer velocity vector:

$$
\begin{aligned}
r= & r_{i}\left[\begin{array}{l}
-p \sin \psi+\sin \left(\psi_{-} \alpha_{i}\right) \\
p \sin \psi_{i}+\sin \left(\psi_{i}-a ;\right)
\end{array}\right]^{\left(p^{2}-1\right) /\left(p^{2}+2 p \cos \alpha_{i}+1\right)} \\
& \times \exp \left[\frac{2 p\left(\psi_{i}-\psi\right) \sin \alpha_{i}}{p^{2}+2 p \cos \alpha_{i}+1}\right]
\end{aligned}
$$

where $a ;=\psi_{i}+\theta_{i}$. We rederive the solution in terms of the LOS angle $\theta$ for consistency with our later derivation.

$$
\begin{aligned}
r= & r_{i}\left[\frac{+p \sin \left(\theta^{+} c\right)}{\sin \theta_{i}+p \sin \left(\theta_{i}+c\right)}\right]^{\left(p^{2}-1\right) /\left(p^{2}+2 p \cos c+1\right)} \\
& \times \exp \left[\frac{2 p\left(\theta_{i}-\theta\right) \sin c}{p^{2}+2 p \cos c+1}\right]
\end{aligned}
$$

Both the solutions for $N=1$ and $N=2$ have the common feature that the angle parameter $\theta$ or $\psi$ cannot be explicitly expressed in terms of $r$. 
To solve for the time $t$, we obtain, from (29)

$$
\begin{array}{r}
\theta=\frac{V_{\theta}(\theta)}{r(\theta)}
\end{array}
$$

whence

$$
\int_{\theta_{i}}^{\theta} \frac{r}{V_{\theta}} d \theta=t-t
$$

The integration here can be performed only for $N=1$, for which case

$$
t=\frac{r_{i} \cos \left(\theta_{i}+p\right)-r \cos (\theta+p)}{p V_{M}-V_{T}}
$$

Equation (34) and (35) jointly relate $r$ and $\theta$ with $\boldsymbol{t}$ and constitute the complete (though implicit) solution for the PPN for a nonmaneuvering target for $N=1$.

The PPN equations have not been solved for higher $N$ values of $3,4,5$, etc., or any other real value, even for the relatively simple case of nonmaneuvering target motion. In the absence of such general solutions, linearized solutions such as those in [8] have hitherto been used to investigate PN behavior for realistic values of $N$. Using such linear analysis, it is also possible to optimize $N$ in the sense of minimum maneuver requirements [8], minimum time until intercept, or other criteria.

Although the complete solution (34) and (38) for $N=1$ and the partial solution (i.e., $r$ as a function of 8 and not of $\boldsymbol{t}$ ) (35) for $\boldsymbol{N}=2$ are exact, they have very limited practical value for the following reasons.

1) They are valid only for the restrictive case of nonmaneuvering targets.

2) The navigation constant $N$ in a general $\mathrm{PN}$ problem is a positive real number which may be optimized with respect to different performance criteria. Forcing it to have discrete values of 1 and 2 would result in grossly nonoptimal performance.

3) Even if one were to choose integer values of $N$, values of 1 and 2 would be too low resulting in sluggish system response and undesirable trajectory behavior. For example $\boldsymbol{N}=\mathbf{1}$ corresponds to pure (or deviated) pursuit course which is known to result in infinite maneuver requirements [7]. $N=\mathbf{2}$ is somewhat better, but the value is still considered too low and leads to singular solutions under certain conditions.

It is clear that the existing special analytical solutions to the PPN problem for the particular case of nonmaneuvering targets are grossly inadequate to handle practical PN problems and there is a definite need for general solutions powerful enough to handle arbitrary values of the navigation constant $N$ for both nonmaneuvering as well as maneuvering targets.

\section{B. Qualitative Analysis of Pure Proportional Navigation}

While, as brought out in the last section, general solutions to the PN trajectory have hitherto not been available, qualitative methods have been used [10-12] to gain a limited insight into the behavior of the differential equations describing the PPN motion. In particular, the qualitative methods have been used to determine conditions under which a pursuer can reach the target from any initial state, and determine bounds on the pursuer acceleration.

It is to be specifically pointed out that the qualitative approach has only provided certain conditions for the PN motion and not solutions, explicit or implicit, for any parameter pertaining to such motion.

1) Nonmaneuvering Target: For rectilinear, constant speed target motion, it was shown by Guelman [10] through a heuristic treatment that if $N V_{M}>V_{M}+V_{T}$ and $V_{M}>V_{T}$, the pursuer will reach the target for all but a finite number of possible initial conditions at launch. For the finite set of initial conditions that satisfy $V_{\theta}\left(\theta_{i}, r_{i}\right)=0$ and $V_{R}\left(\theta_{i}, r_{i}\right)>0$, i.e., the initial geometry is such that a collision course results but the pursuer is going away from the target, the pursuer will not reach the target. Thus, if $V_{M}>V_{T}$, for all values of $N>2$ and under all normal initial conditions, the pursuer will always reach the target.

Also, it was proved in the same paper that, if $V_{M}>V_{T}$ and $((N-2) / 2) V_{M}>V_{T}$, the rate of rotation of the LOS decreases in the final phases of the pursuit. It may be noted that for $N>4$ this condition is always fulfilled. If $N<4$, then this condition serves to determine the minimum value for $N$, i.e., $N>$ $2\left(V_{M}+V_{T}\right) / V_{M}$.

In a later work, Guelman [12] demonstrated that the normal acceleration of a pursuer engaging a nonmaneuvering target is a uniformly decreasing function of time if $V_{M}>\sqrt{2} V_{T}, N>4$ and initially the pursuer is closing toward the target.

2) Maneuvering Target: For targets with lateral acceleration, Guelman [12] showed that if $V_{M}>\sqrt{2} V_{T}$ and $N V_{M}>V_{M}+V_{T}$, then the pursuer reaches the target for all initial launch conditions except for those where the initial engagement geometry is such as to make $\dot{\theta}_{i}=0$ and the pursuer is moving away from the target.

Also, if initially the pursuer is closing toward the target with $V_{M}>\sqrt{2} V_{T}, N>4$ and the initial lateral acceleration $A_{M I}$ of the pursuer is such that a) if $\left|A_{M i}\right|>A_{M m}$ then $\left|A_{M}\right|$ will decrease until $\left|A_{M}\right| \leq$ $A_{M m}$, and b) if $\left|A_{M i}\right| \leq A_{M m}$ then $\left|A_{M}\right| \leq A_{M m}$ where $A_{M m}=[N /(N-4)]\left[V_{M} / V_{T}\right]\left|A_{T}\right|$.

\section{Classical Linear Solutions of Pure Proportional Navigation}

In the absence of general closed-form solutions of the nonlinear PPN equations, various forms of linearization have been resorted to. These are based on the assumption that the engagement geometry stays close to a collision course with a small LOS angle and 
the target heading $\beta$ does not change much from its initial direction $\beta_{i}$. Such assumptions lead to a linear time-varying differential equation of first order for the LOS rate (nonhomogeneous for maneuvering target and homogeneous for nonmaneuvering target) which is straightforward to solve [8]. The resulting linear solutions are quite accurate for small values of 6, $\Delta \phi_{i}$, and $A_{T}$ (i.e., a near-tail-chase geometry with a trajectory close to the collision course and small target maneuvers), but becomes increasingly inaccurate for larger values of $\mathbf{6}, \Delta \phi_{i}$, and $\boldsymbol{A} \boldsymbol{T}$, becoming rather unacceptable for large values of these parameters [13].

\section{Quasilinear Solutions of Pure Proportional Navigation}

The severe restrictions on the engagement geometry and the target maneuver levels, necessary to ensure the validity of classical linear solutions, have been to a large extent obviated by adopting a quasilinear approach $[15,16]$. Although such an approach is normally used for numerical iteration, by confining attention only to the terms up to the first derivative and considering only one iteration, it is possible to obtain analytical expressions for all the trajectory parameters for both nonmaneuvering as well as maneuvering targets. Such a solution has the merit of providing accurate analytical estimates of the trajectory parameters for engagement geometries that are significantly far from the tail-chase and for high target maneuver levels.

\section{TRUE PROPORTIONAL NAVIGATION}

In TPN the commanded acceleration is applied normal to the LOS. TPN first appeared in open literature in an article by Murtaugh and Kriel [9] where a linearized treatment was used to obtain the solution for trajectory parameters for a nonmaneuvering target. The resulting solutions obtained were similar to those obtained for the PPN case.

The equations of motion under TPN can be written using (6) and (11) by equating the components of $A_{M}$ and $d^{2} \mathbf{r} / d t^{2}$ along $\boldsymbol{e}$, and $\mathbf{e}_{\theta}$, respectively

$$
\ddot{r}-r \ddot{\theta}^{2}=0
$$

and

$$
r e+2 r e=-c e
$$

where $C=-\lambda V_{R i}$.

\section{A. Closed-Form Solution}

A close look at the system of equations (39) and (40) reveals that since the right-hand side (RHS) of

(39) equals zero, it is possible to obtain an equation in $r$ only which can then be solved. Dividing (40) by $r \dot{\theta}$ we obtain

$$
\frac{\overline{\boldsymbol{\theta}}}{\boldsymbol{\sigma}}+2 \frac{\mathbf{r}}{r}+\frac{\mathbf{c}}{r}=0
$$

Integrating (41) we get

$$
\dot{\theta}=K r^{-2} \exp \left[-N \int \frac{d t}{r}\right]
$$

where $K$ is the constant of integration. Substituting the value of $\dot{\theta}$ in (39) we obtain

$$
r^{3} \ddot{r}=K^{2} \exp \left[-2 N \int \frac{d t}{r}\right] .
$$

Equation (43) can also be rearranged to give the value of the integral

$$
\exp \left[-N \int \frac{d t}{r}\right]=r^{3} \stackrel{\dot{y}}{K^{2}} .
$$

Differentiating (43) and then using the value of the integral from (44), we obtain

$$
r \dot{r}+(3+2 C) \ddot{r}=0 .
$$

Equation (45) was originally obtained by Guelman [3], but the approach followed in this work is more direct and straightforward.

Equation (45) is in terms of $r$ only and is open to solution in terms of that variable. Guelman [3] has obtained such a solution in the closed form, though the complex nature of the problem has necessitated multiple changes of variable, leading to some loss of physical insight, and the resulting solutions are implicit in terms of these indirect variables.

It is to be noted here that a univariate form such as (45), leading to a closed-form solution, has been possible only because the RHS of (39) is zero, as in the case of nonmaneuvering targets. Such neat manipulation is not possible if the RHS of (39) is nonzero which would be the case for a maneuvering target and/or further generalizations of TPN.

This approach to the closed-form solution of the TPN problem, which is the only one available yet, therefore leads to a dead-end solution which is valid only for TPN applied to nonmaneuvering targets, but cannot be generalized further either in terms of target motion or in terms of the PN scheme.

It is obvious from the definitions of TPN and PPN that they would both reduce to the collision course as the heading error vanishes; therefore it is not surprising that the behavior of the TF' solution is similar to that of the PPN when the engagement geometry is close to a collision course. However, for geometries significantly different from the collision course, $\mathbf{T F}$ ' shows remarkably different behavior as compared with PPN. In particular, the Occurrence of intercept in case of TPN is restricted to engagements where the initial conditions lie within a determined circle, defined as the circle of capture. This is usually 
far more restrictive than the case of PPN for which capture is assured for all initial pursuer directions except for a precisely defined particular direction (Section IVB1 and IVB2) as long as the following liberal conditions are satisfied: 1) for nonmaneuvering target: $V_{M}>V_{T}$ and $N V_{M}>V_{M}+V_{T}$, and 2) for laterally maneuvering target: $V_{M}>\sqrt{2} V_{T}$ and $N V_{M}>$ $V_{M}+V_{T}$. These conditions are always satisfied if $N>$ 2 which is the case for most normal PN engagements. In addition, since a component of the control force is applied along the pursuer velocity vector in the case of TPN, this results in forward speed variations of the pursuer. Such is not the case with PPN.

Thus, although a closed-form solution is available for TPN for the nonmaneuvering target case, the TPN law itself is of limited practical value due to the following reasons: 1) TPN imposes severe restrictions on the initial launch geometry in order to ensure intercept of the target even for the commonly used values of the navigation constant $(\lambda>3)$. 2) The control effort tends to vary the pursuer forward velocity throughout the engagement which is not possible with the commonly used aerodynamic controls; for reaction control, it necessitates thrusters with forward acceleration/deceleration capability.

\section{GENERALIZEDTRUE PROPORTIONAL NAVIGATION}

In the last Section, it was pointed out that although the TPN case resulted in a closed-form solution, at least for nonmaneuvering targets, one of the major weaknesses of TPN is the limited capture area, which severely restricts the choice of the initial launch conditions to ensure intercept. In a recent article [4], a GTPN law has been proposed which claims to minimize this drawback. In GTPN, the pursuer acceleration is not necessarily applied normal to the LOS, but maintains a fixed angle y with respect to the normal to the LOS. TF" is a special case of GTPN when $\mathrm{y}=0$.

The equations of motion under GTPN can be written using (6) and (9) by equating the components of $A_{M}$ and $d^{2} \mathbf{r} / d t^{2}$ along $\mathbf{e}_{r}$ and $\mathrm{e}_{\theta}$, respectively

$$
\ddot{r}-r \ddot{\theta}^{2}=\lambda V_{\theta i} \sin \gamma
$$

and

$$
r \ddot{\theta}+2 \dot{r} \dot{\theta}=-\lambda V_{R i} \cos \gamma
$$

As discussed in the last section, the method used to solve the TPN case cannot be used to solve the above system of equations (46) and (47) representing the GTPN case. To solve these equations, the independent variable is changed to 8 . Also, the system of equations governing the pursuer motion are written in terms of $V_{R}$ and $V_{\theta}$. The system of these first-order differential equations is then solved to give $V_{R}$ and $V_{\theta}$ in terms of the LOS angle 8. This solution is then used to obtain the inequality defining the capture area (i.e., the relationship between $V_{R i}, V_{\theta i}, \lambda$, and y which satisfy the constraints $V_{\theta}\left(\theta_{f}\right)=0$ and $\left.V_{R}\left(\theta_{f}\right)<0\right)$. The capture area so obtained is not necessarily a circle. Next, a differential equation relating the angular momentum $h\left(=r V_{\theta}\right)$ and $\theta$ is obtained. The solution of this equation, involving the evaluation of a complicated integral, provides $h$ in terms of 8 . However, the integral can be evaluated, in terms of elementary or elliptic functions, only if the RHS of (46) and (47) are trigonometric functions. Once the solution for $h$ has been found, the LOS rate $\dot{\theta}$ can be obtained as $\dot{\theta}=V_{\theta}^{2} / h$. The relative distance $r$ is found as $r=h / V_{\theta}$ and the duration $t_{f}$ of pursuit is obtained by a further integration of the expression for $\boldsymbol{r}$ within the limits of 8 ; and $\theta_{f}$, where $\theta_{f}$ is obtained by solving the transcendental equation $V_{\theta}\left(\theta_{f}\right)=0$.

The solution obtained for GTPN using the above methodology is implicit in nature. Although an inequality defining the capture area is obtained in [4], no explicit expressions for any of the trajectory parameters such as $8, \dot{\theta}, A_{M}$ or time $t$ are presented.

It has been established in [4] that GTPN has a larger capture area than TPN in the region where $C=\left|V_{R i} / V_{\theta i}\right|^{1 / 2}$ is small. Also, $t_{f}$ is very sensitive to variations in $\mathrm{y}$, for small and large values of $\mathrm{y}$, and is relatively invariant for moderate values of $\gamma$. However, it has been observed that for slightly large values of $\gamma$, where the advantage of larger capture area is claimed, GTPN results in unbounded LOS rate and control force even for normal values of navigation constant such as $\lambda=3$. Thus, the advantage of slightly larger capture area in the case of GTPN is greatly offset by the highly undesirable trajectory behavior.

Since GTPN is a generalization of TPN, the method used to solve GTPN can also be used to solve for TPN as a special case. GTPN is in turn a special case of a more general guidance law called the Generalized Guidance Law [6] in which the applied control effort is made proportional to the rate of change of a generalized vector $\mathbf{L}$ in a two-dimensional space. The generalized direct in $\mathbf{L}$ is written as

$$
\mathbf{L}=f(r, \theta) \mathbf{e}_{r}+g(r, \theta) \mathbf{e}_{\theta} .
$$

The Generalized Guidance Law requires that the pursuer acceleration to be commanded should be equal to the time rate of $\mathbf{L}$, i.e.,

$$
\begin{aligned}
A_{M}= & \mathrm{L} \\
= & \left(\frac{\partial f}{\partial r} \frac{d r}{d \theta}+\frac{\partial f}{\partial \theta}-g\right) \dot{\theta} \mathbf{e}_{r} \\
& +\left(\frac{\partial g}{\partial r} \frac{d r}{d \theta}+\frac{\partial g}{\partial \theta}+f\right) \dot{\theta} \mathbf{e}_{\theta} .
\end{aligned}
$$

It is easily seen that depending upon the choice of $f(r, \theta)$ and $g(r, \theta)$ a family of LOS-referenced guidance laws will result. In particular, if $f(r, \theta)=\lambda$ 


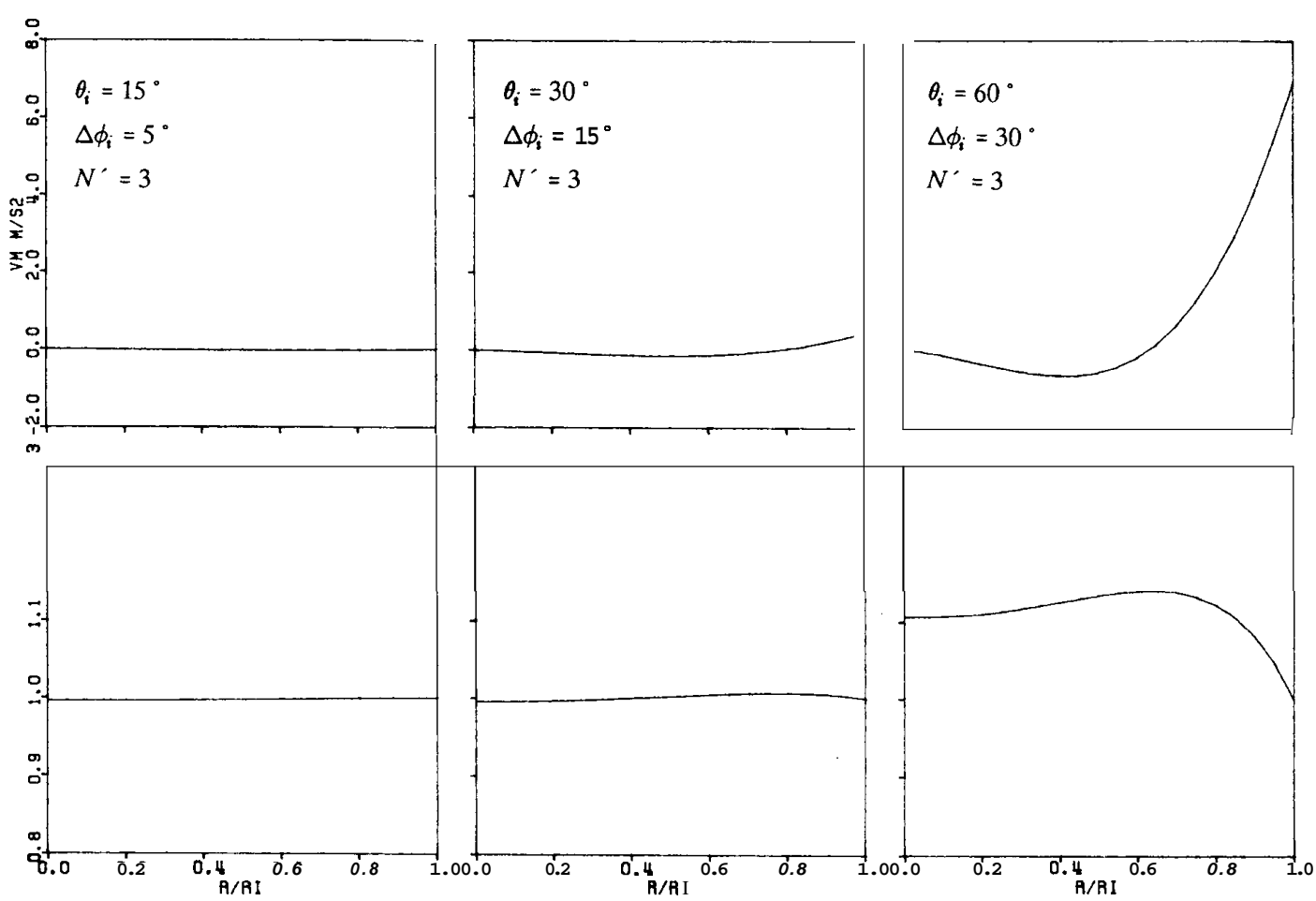

Fig. 4. Variation of $V_{M}$ and $\dot{V}_{M}$ as function of $r / r_{i}$ for TPN. For PPN, $\dot{V}_{M}=0$ and $V_{M} / V_{M i}=1$ for all values of $r / r_{i}$.

and $g(r, \theta)=0$ then TPN results, and $f(r, \theta)=$ Xcosy and $g(r, \theta)=\lambda \sin \gamma$ would result in GTPN.

Although the Generalized Guidance Law is indeed quite general as far as LOS-referenced guidance laws go, the integral involved in obtaining the solution for the differential equation in $h$ and $\theta$ is shown [6]to be tractable for the particular cases of TPN, GTPN, and Prediction Guidance Law [14] and that too only for the case of nonmaneuvering target. However, it is easy to see that since the PPN control force is referenced relative to the pursuer velocity vector which has no fixed angular relationship with the LOS, LOS-referenced guidance laws such as the Generalized Guidance Law and its special cases cannot include the PPN, i.e., the PPN is not a natural candidate for definition under the Generalized. Guidance Law. As such, the relatively considerable success achieved in the analysis of LOS-referenced guidance laws cannot be made use of in treating the PPN problem.

\section{CRITICAL COMPARISON BETWEEN DIFFERENT TYPES OF PN LAWS}

The aim of this section is to present a comparative study of the PPN, TPN, and GTPN guidance laws with regard to their definition, implementation, analytical aspects including the method and the nature of solution, and more importantly, an appraisal of the behavior of the pursuer motion resulting from these guidance laws. These guidance laws have been individually discussed in the preceding sections.

\section{A. Forward Velocity Variation}

In PPN the commanded acceleration is applied normal to the pursuer velocity vector; thus there is no component of acceleration along the pursuer heading direction and hence the pursuer forward velocity $V_{M}$ remains constant. In contrast in TPN, the commanded pursuer acceleration is applied normal to the LOS, resulting in acceleration both along and normal to the pursuer velocity vector. The forward acceleration component is given as $\dot{V}_{M}=-A_{M} \sin (\theta-\phi)$.

In GTPN, the pursuer acceleration is applied at a fixed angle $y$ with the normal to the LOS. Here again, in general, the commanded acceleration has components both along and normal to the pursuer velocity vector. The forward acceleration component here is given as $\dot{V}_{M}=-A_{M} \cos \gamma \sin (\theta-\phi)$.

A plot showing the variation of $\dot{V}_{M}$ as a function of the normalized range $r / r_{i}$ is shown in Fig. 4 for the case of TPN. These plots have been obtained by numerical integration of the original equations of motion in Section V. Similar plots for PPN would be straightforward, being uniformly equal to zero. The $\dot{V}_{M}$ graphs in Fig. 4 corroborate the fact that the TPN scheme requires considerable acceleration/deceleration along the flight direction of the pursuer. This effect 
TABLE I

Cumulative Velocity Increment A V Indicating Total Control Effort for TPN and PPN

\begin{tabular}{|c|c|r|c|c|c|c|c|}
\hline \multirow{3}{*}{$\theta$} & \multirow{2}{*}{$\Delta \phi_{\mathrm{i}}$} & \multicolumn{6}{|c|}{$\Delta \mathrm{Vm} / \mathrm{s}$} \\
\cline { 3 - 9 } & & \multicolumn{2}{|c|}{$\mathrm{N}=\mathbf{2}$} & \multicolumn{2}{|c|}{$\mathrm{N}=3$} & \multicolumn{2}{c|}{$\mathrm{N}=\mathbf{4}$} \\
\cline { 3 - 9 } & & $\mathrm{TPN}$ & $\mathrm{PPN}$ & $\mathrm{TPN}$ & $\mathrm{PPN}$ & $\mathrm{TPN}$ & $\mathrm{PPN}$ \\
\hline \hline \multirow{3}{*}{30} & $\mathbf{1 5}$ & $\mathbf{4 3 . 7 6}$ & $\mathbf{4 2 . 8 8}$ & $\mathbf{3 3 . 1 5}$ & $\mathbf{3 2 . 8 0}$ & $\mathbf{2 9 . 0 7}$ & $\mathbf{2 8 . 7 2}$ \\
$\mathbf{6 0}$ & $\mathbf{4 0}$ & $\mathbf{1 2 3 . 6 7}$ & $\mathbf{9 6 . 8 2}$ & $\mathbf{9 2 . 3 4}$ & $\mathbf{7 9 5 8}$ & $\mathbf{8 0 . 9 5}$ & $\mathbf{7 1 . 6 8}$ \\
\hline
\end{tabular}

Note: Roman letter symbols here correspond to italic letter symbols in text.

is very pronounced for large departures of the geometry from the collision course. Such forward acceleration/deceleration are impossible to apply in a controlled manner using aerodynamic/hydrodynamic control surfaces which are most frequently used in PN applications. With reaction type control, which is normally adopted outside the atmosphere, such acceleration/deceleration is possible but cumbersome and, as shown below, wasteful.

The cumulative effect of the longitudinal acceleration/deceleration is to cause forward velocity variations of the pursuer as shown in the normalized $V_{M}$ plots for TPN in Fig. 4. These plots have been obtained by numerical integration of the original equations of motion in Section V. Similar plots for PPN are straightforward, being constant at a value of unity. Such pronounced speed variations for TPN would result in changes in the aerodynamic characteristics, control system behavior, and autopilotage.

The kinematic control efficiency of the two PN schemes under discussion, the PPN and the TPN, is tabulated in Table I. The control efficiency is indicated by the integral of the magnitude of the lateral acceleration, i.e., $\int_{0}^{t_{f}}\left|A_{M}\right| d t$ [9]. Table I shows that PPN consistently requires a lower control effort than TPN for all geometries and navigation constant values. As expected, the difference is small for shallow geometries, i.e., those close to tail-chase and collision course, for which conditions both the PN schemes show similar behavior, as discussed earlier. However, at larger departures from tail-chase and the collision course conditions, the disadvantage of TPN is very pronounced, reaching a value as much as $\mathbf{2 7 . 7}$ percent (of extra control effort requirement relative to PPN) for $\theta_{i}=60^{\circ}, \Delta \phi_{i}=40^{\circ}$, and $N^{\prime}=2$. The relative inefficiency of the TPN can be directly traced to the longitudinal acceleration/deceleration caused by the TPN scheme. Such acceleration/deceleration does not contribute to the lateral maneuver of the pursuer, but consumes control effort nevertheless.

From the foregoing discussions, it is clear that the TPN law is practically difficult to implement in most commonly used present day aerodynamically controlled missiles. It may find application only in space vehicle guidance but even in this case the TPN law results in inefficient navigation.

\section{B. Analytical Aspects}

As discussed earlier in Section $\mathbf{V}$, the method used to obtain the solution for pursuer motion under TPN is a special one, applicable only to nonmaneuvering targets. It cannot be extended to the case of maneuvering targets or to further generalizations of TPN. Even for the restricted case of nonmaneuvering target, the solution requires a sequence of variable transformations resulting in loss of physical insight.

The GTPN case has been solved by adopting the LOS angle $\theta$ as the independent variable. The solutions thus obtained are implicit in nature. Although an expression for the inequality representing the capture region is available, no explicit solutions for any of the trajectory parameters have yet been possible. Also, the general solution of the GTPN involves an integral which has been integrable only for the case of nonmaneuvering targets and even that only for relatively simple TPN variants.

In contrast to TPN and its generalizations, the PPN equations have generally proven more difficult to solve. Thus, the exact solutions available for PPN are much more restricted than for TPN and its generalizations, covering only the nonmaneuvering target case and the discrete values of 1 and 2 of the navigation constant $N$.

\section{Trajectory Behavior}

TPN and GTPN are special cases of a Generalized Guidance Law, where the pursuer acceleration is proportional to the derivative of a general direction vector $\mathbf{L}$ in a two-dimensional space [6]. Although this class of guidance laws are mathematically tractable, at least for the nonmaneuvering target case, the laws themselves give rise to undesirable trajectory behavior such as restricted capture area, unbounded acceleration, etc. These are discussed in the following paragraphs.

1) Capture Area: For the TPN case, the capture area is delineated by the inequality [3]

$$
\left(V_{R i}+C\right)^{2}+V_{\theta i}^{2}<C^{2} \text {. }
$$

This is a circle centered at $(-C, 0)$ with radius $C$ and is depicted in [3, Fig. 3]. The center of the circle corresponds to $\lambda=\mathbf{1 . 0}$ while the bottom end of the circle $(-2 C, 0)$ corresponds to $\lambda=0.5$.

For GTPN, the capture area is defined by [4]

$$
\left|\frac{V_{R i}}{V_{\theta i}}\right|^{2}<-\frac{1-2 C \sin \gamma}{1-2 C \cos \gamma} .
$$

The total capture area is characterized by the RHS 
region of the contour defined by the equation

$$
C=\frac{\left(V_{R i} / V_{\theta i}\right)^{2}+1}{2 \sqrt{\left(V_{R i} / V_{\theta i}\right)^{4}+1}}
$$

and is depicted in $[\mathbf{4}$, Fig. 1]. It can be seen that GTPN has a larger capture area than that of TPN $(\mathrm{y}=0)$ in the region where $\left|V_{R i} / V_{\theta i}\right|$ is small.

The capture behavior of TPN and GTPN may be contrasted with the PPN case where intercept is always possible except for one definite direction when $V_{\theta i}=0$ and $V_{R i}>0$ provided $V_{M}>V_{T}$ and $N V_{M}>V_{M}+V_{T}$; this condition being always satisfied for $N>2$, as is usually the case. Thus, whereas capture is always possible under the PPN scheme for all commonly used values of navigation constant and launch geometries, TPN and GTPN impose great restrictions on the initial conditions for intercept to occur.

2) Boundedness of Acceleration/LOS Rate: As discussed earlier in Section IVB1), the PPN pursuer lateral acceleration is a uniformly decreasing function of time if $V_{M}>\sqrt{2} V_{T}$ and $N>\mathbf{4}$.

For TPN, the pursuer lateral acceleration is a uniformly decreasing function of time only if $\lambda+$ $\left(V_{\theta i} / V_{R i}\right)^{2}>3$. This again imposes restrictions on initial conditions for the LOS rate to remain bounded and is shown in [3, Fig. 6].

However, perhaps the worst behavior with regard to the boundedness of the LOS rate is shown by GTPN, where, even for the normal values of the navigation constant $\lambda$, the LOS rate becomes unbounded as $y$ increases from 0 to slightly larger values. The behavior is depicted in $[4$, Fig. 3] for $\lambda=\mathbf{3}$.

Thus, whereas the LOS rate (to which lateral acceleration is proportional) remains bounded throughout the engagement for PPN for all commonly used values of the navigation constant, TPN again imposes restrictive conditions on the initial geometry. In the case of GTPN, as the angle y increases from 0 (TPN case) to larger values, the LOS rate becomes unbounded even for the normally used values of navigation constant. It may be noted that GTPN [4] has claimed superiority over TPN in terms of larger capture area precisely when the angle $\gamma$ is large.

3) Robustness: From the preceding discussions it is obvious that the TPN, GTPN, and their generalizations are not robust in the sense that desirable trajectory behavior is strongly dependent on the initial engagement geometry and even intercept is not assured under all engagement conditions. Thus, the practical usefulness of these laws is highly restricted, since establishing favorable initial conditions may not always be possible for all engagement scenarios.

\section{Summary of Comparative Features}

In brief, PN laws referenced to the LOS (such as TPN, GTPN, and Generalized Guidance Law) are impractical due to the following reasons.
1) Requirement of thrusters to provide for forward velocity variation; implementation not possible in aerodynamically controlled pursuers.

2) Inefficiency of control effort relative to PPN.

3) Restriction on initial conditions so as to ensure intercept.

4) Unboundedness of acceleration, especially for GTPN.

$5)$ Lack of robustness.

From a practical point of view, PPN (and other similar laws referenced to the pursuer velocity vector) is a guidance law far superior to TPN and other LOS-referenced guidance laws. From this viewpoint, the only utility of the latter class of laws appears to be that they can serve as an approximation to the more practical PPN law and yet provide analytical solution under somewhat broader conditions than is possible for PPN. Clearly, the approximation of PPN by TPN is valid only for geometries close to the collision course (Section V), for which case linear solution itself gives reasonably accurate results. For the more general geometries, there is no alternative but to solve the PPN problem.

\section{CONCLUSIONS}

In this paper two generic classes of PN laws have been compared in detail. One class consists of pursuer velocity-referenced systems which include PPN and its variants and the second category consists of LOS-referenced systems such as TPN, GTPN, and the Generalized Guidance Law. It has been established that in spite of some restricted advantages in solvability of the equations of motion, the LOS-referenced PN schemes suffer from serious limitations in terms of implementation and trajectory behavior. Among the major drawbacks are forward velocity variation requirement (i.e., forward acceleration and braking), relatively large control effort requirement, restrictions on initial conditions to ensure intercept, lack of robustness, and possibility of unbounded acceleration. This leads to the firm conclusion that PPN is the more natural guidance law in a practical sense compared with TPN and its generalizations.

Thus, although more analytical effort appears to have been bestowed on TPN and its generalizations, the authors contend here that more serious efforts need to be made to understand, model, and solve the PPN guidance problem. The authors have made a contribution in this direction through their quasilinearized approach to the solution of the PPN problem which has been shown to be far more accurate and general in terms of geometries and target maneuver levels handled. In view of the great practical importance of PPN over TPN and its generalizations, further efforts are called for in understanding the PPN as a guidance law. 


\section{REFERENCES}

[1] Siouris, G. M., and Leros, P. (1988)

Minimum-time intercept guidance for tactical missiles. Control Theory and Advanced Technology, 4, 2 (1988), 251-263.

[2] Nesline, E W., and Zarchan, P. (1979)

A new look at classical versus modern homing missile guidance.

In AIAA Guidance and Control Conference Proceedings, 1979, 230-242.

[3] Guelman, M. (1976)

The closed form solution of true proportional navigation. IEEE Transactions on Aerospace and Electronic Systems, AES-7, 4 (July 1976), 472-482

[4] Yang, C. D., Yeh, E B., and Chen, J. H. (1987)

The closed form solution of generalized proportional navigation.

Journal of Guidance, Control and Dynamics, 10, 2 (Mar.-Apr. 1987), 216-218.

[5] Yang, C. D., and Yeh, F. B. (1987)

Closed form solution for a class of guidance laws.

Journal of Guidance, Control and Dynamics, 10, 4 (Aug. 1987), 412-415.

[6] Yang, C. D., Yeh, E B., and Hsiao, E B. (1989)

Generalized guidance law for homing missiles. IEEE Transactions on Aerospace and Electronic Systems, AES-25, 2 (Mar. 1989) , 197-212

[7] Locke, A. S. (1956)

Guidance.

Princeton, N J Van Nostrand, 1956.

[8] Jerger, J. J. (1960)

Systems Preliminary Design.

Princeton, NJ: Van Nostrand, 1960.
[9] Murtaugh, S. A., and Criel, H. E. (1966)

Fundamentals of proportional navigation.

IEEE Spectrum, 3, 12 (Dec. 1966), 75-85.

[10] Guelman, M. (1971)

A qualitative study of proportional navigation.

IEEE Transactions on Aerospace and Electronic Systems, AES-7, 4 (July 1971), 337-343.

[11] Guelman, M. (1972)

Proportional navigation with a maneuvering target. IEEE Transactionson Aerospace and Electronic Systems, AES-8, 3 (May 1972), 364-371.

[12] Guelman, M. (1973)

Missile acceleration in proportional navigation. IEEE Transactions on Aerospace and Electronic Systems, AES-9, 3 (May 1973), 462-463.

[13] Shukla, U. S. (1988)

New kinematic studies on the proportional navigation problem.

Ph.D. dissertation, Department of Aerospace Engineering, Indian Institute of Science, Bangalore, India, Sept. 1988.

[14] Kim, Y. S., Cho, H. S., and Bien, Z (1985)

A new guidance law for homing missiles.

Journal of Guidance, Control and Dynamics, 8, 3 (May-June 1985), 402-404

[15] Shukla, U. S., and Mahapatra, P. R. (1988) A generalized linear solution of proportional navigation. IEEE Transactions on Aerospace and Electronic Systems, AES-24, 3 (May 1988), 231-238.

[16] Mahapatra, P. R., and Shukla, U. S. (1989)

Accurate solution of proportional navigation for maneuvering targets.

IEEE Transactions on Aerospace and Electronic Systems, AES-25, 1 (Jan. 1989), 81-89.

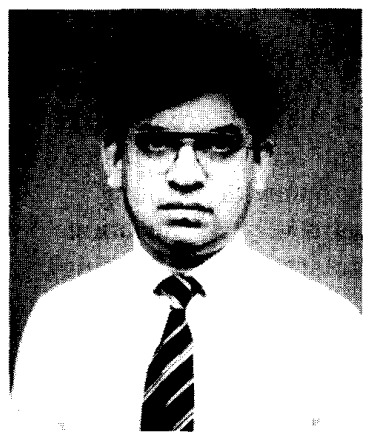

Uday S. Shukla was born in 1951 in Mathura (U.P.), India. He received the Bachelor's degree in electrical communication engineering in 1973 and the Ph.D. degree in aerospace engineering in 1989, both from the Indian Institute of Science, Bangalore, India.

Until recently, he was employed with Hindustan Aeronautics Limited in Bangalore, working on avionics and navigation systems. Currently, Dr. Shukla is with the Centre for Development of Advanced Computing in Bangalore, looking after system software development for parallel machines.

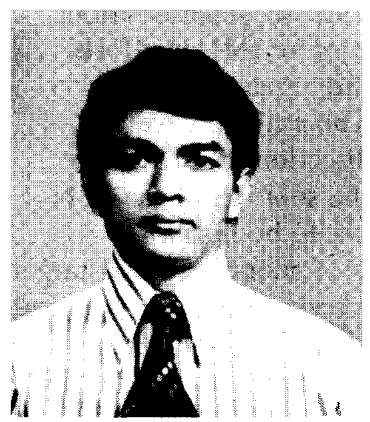

Pravas R. Mahapatra received the B.Sc. (Engg) from Regional Engineering College at Rourkela, and the M.E. and Ph.D. degrees from the Indian Institute of Science at Bangalore, India.

Dr. Mahapatra has been teaching at the Department of Aerospace Engineering in the Indian Institute of Science since 1970, where he is currently an Associate Professor. He has a broad area of active interest within the field of aerospace and electronic systems. This includes radar systems, navigational aids, and navigation theory and flight safety aspects with particular reference to weather phenomena and air traffic control. Dr. Mahapatra is a member of the U.S. Institute of Navigation. 